\title{
CHARGED VORTICES IN THE NEGATIVE U HUBBARD MODEL
}

\author{
L. F. FEINER $\left(^{*}\right)$ and J. ZAANEN $\left(^{* *}\right)$
}

${ }^{*}$ ) Philips Research Laboratories, P.O.Box 80000, 5600 JA Eindhoven, The Netherlands

(**) Max Planck Institut für Festkörperforschung, Heisenbergstrasse 1, 7000 Stuttgart 80, F.R.G.

We have studied the negative $U$ Hubbard model as a candidate generic model for high- $T_{\mathrm{c}}$ superconductivity with short coherence length. We show that in the strong coupling limit the structure of the vortex is qualitatively different from the usual picture. Charge is accumulated in the vortex and the core is insulating, while the size depends on the band filling.

\section{INTRODUCTION}

Evidence has been accumulating that the superconducting state in the high $T_{\mathrm{c}}$ superconductors is rather unusual. There are strong indications that it is of strong coupling nature, and the proportionality between hole count and $T_{\mathrm{c}}$ points at instantaneous interactions, suggesting a breakdown of the Migdal approximation. In this context the negative $U$ Hubbard model has recently $1,2,3,4$ drawn renewed attention. Here, in the strong coupling limit the coherence length (i.e. spatial extent of the Cooper pairs) becomes of the order of a lattice spacing, as appropriate for the conditions just sketched.

In the present paper we show that in this model the nature of the vortex is unusual for superconductivity and in fact reminiscent of superfluidity. The structure of the vortex is determined by the energy balance that arises from the coupling between particle density and superconductive order. Consequently, charge accumulates in the vortex core and the total charge of an isolated vortex diverges. Further, the vortex core is insulating and the size of the vortex is inversely proportional to band filling.

\section{STRONG COUPLING NEGATIVE U HUBBARD MODEL}

In the strong coupling limit the negative $U$ Hubbard model is equivalent to a spin $1 / 2$ model ${ }^{5}$ :

$H=J \sum_{i, j}\left[S_{i}^{z} S_{j}^{z}-\left(S_{i}^{x} S_{j}^{x}+S_{i}^{y} S_{j}^{y}\right)\right]-B \sum_{i}\left(2 S_{i}^{z}+1\right),(1)$

with spin up (down) corresponding to a doubly occu- pied (empty) site. Here $J=2 t^{2} /|U|$ and $B=\mu+|U| / 2$. In mean field (MF) approximation, away from half filling the low temperature phase is a superconducting (SC) phase (a spin flop phase in spin language) with order parameter $\left\langle c_{i \uparrow} c_{i\rceil}^{\dagger}\right\rangle=$ $\left\langle S^{+}\right\rangle=\left\langle S_{\perp}\right\rangle \exp (i \varphi)$, while the average particle number is given by $n=2\left\langle S^{z}\right\rangle+1=\cos \theta+1$. At $T=0$ one has $2\left\langle S^{z}\right\rangle=\cos \theta_{0}=B / z J, z$ being the number of neighbors, and $2<S_{\perp}>=\sin \theta_{0}$. In $\mathrm{MF}$ the phase $\varphi$, although arbitrary, is fixed, thus breaking the symmetry in the xy-plane.

We demonstrate here that the MF equations also allow for nonuniform solitonlike solutions which represent topological defects. The interesting feature is that they necessarily involve spatial variation of both $\left\langle S^{z}\right\rangle$ and $\left\langle S_{\perp}\right\rangle$, i.e. of both particle density and superconducting order parameter. The reason is that in the present model it is assumed that the interactions are large compared to the bandwidth ('ultra-strong coupling') while there is no frequency cut-off ('instantaneous interactions'). As a consequence all carriers participate in the SC condensate. This partially restores the symmetry between diagonal and offdiagonal charge density: if the particle density (or hole density for a more than half filled band) increases, $T_{\mathrm{c}}^{*}$ increases, and if the $\mathrm{SC}$ order is weakened as in the vortex, the particle (or hole) density decreases.

We have restricted ourselves to $T=0$, which should be sufficient to bring out the essential structure of the solitons. We have investigated the solitons both by numerical solution of the MF equations on a finite 
lattice and by analytic treatment of the continuum limit. In the quasi-1D case we find charged domain walls ${ }^{6}$; here we present results on the $2 \mathrm{D}$ vortex.

\section{VORTICES}

Because of the nearly $2 \mathrm{D}$ nature of the superconductivity associated with the $\mathrm{CuO}_{2}$-planes in the high- $T_{\mathrm{c}}$ oxides, the vortices that occur in $2 \mathrm{D}$ are of special interest, in particular since they might give rise to a Kosterlitz-Thouless phase instead of true long range order 4 . Our numerical results for square lattices with up to $40 \times 40$ sites and antiperiodic boundary conditions indeed show stable vortex solutions with $\left.2<S^{z}\right\rangle$ going to 1 in the centre. So charge accumulates in the vortex core, as illustrated in the cross sections shown in fig. 1 .

The origin of this behavior is readily recognized from the continuum limit, where the energy density is

$$
\begin{aligned}
E=[ & \frac{1}{2} z J \cos 2 \theta|\nabla \theta|^{2}+\frac{1}{2} z J \sin ^{2} \theta|\nabla \varphi|^{2} \\
& +2(z J \cos 2 \theta-4 B \cos \theta-4 B)] / 8 a^{2} .
\end{aligned}
$$

For a simple vortex $\varphi(r, \phi)=\phi$ (using polar coordinates $r, \phi)$ one has $|\nabla \varphi|=1 / r$, and the resulting divergence in (2) is then suppressed by having $\theta(r) \rightarrow 0$ linearly in $r$ as $r \rightarrow 0$. Actually, one easily verifies that one of the two Euler equations associated with (2) is solved by the above $\varphi(r, \phi)$, but that a constant $\theta(r)$ is then not a solution of the remaining one.

Particle density and SC order parameter approach their asymptotic values not exponentially but according to a power law: $\cos \theta \approx \cos \theta_{0} /\left(1-1 / 8 r^{2}\right)$ and $\sin \theta \approx \sin \theta_{0}\left(1-\left[2 / 8 r^{2}\right)\right.$ for large $r$, where $l=a \mid \tan \theta_{0}$. For $n \gtrsim 1.5$ these expressions describe the numerical results within 1 percent after a few lattice spacings from the vortex center. The size of the vortex is seen to diverge as $1 / \sqrt{n(2-n)}$ as the band becomes completely empty or completely full.

For a single vortex the energy shows the characteristic logarithmic divergence with system size, $E_{\mathrm{v}}=E_{\mathrm{c}}+(\pi J / 2) \sin ^{2} \theta_{0} \ln \left(R / R_{\mathrm{c}}\right)$, where $R_{\mathrm{c}}$ and $E_{\mathrm{c}}$ are a core radius and core energy. The vortex charge diverges as well, since the number of particles contained in the vortex is $N_{\mathrm{v}}=N_{\mathrm{c}}+$ $(\pi / 4) \cos \theta_{0} \ln \left(R / R_{\mathrm{c}}\right)$. However, for a vortexantivortex pair both energy and charge are finite.

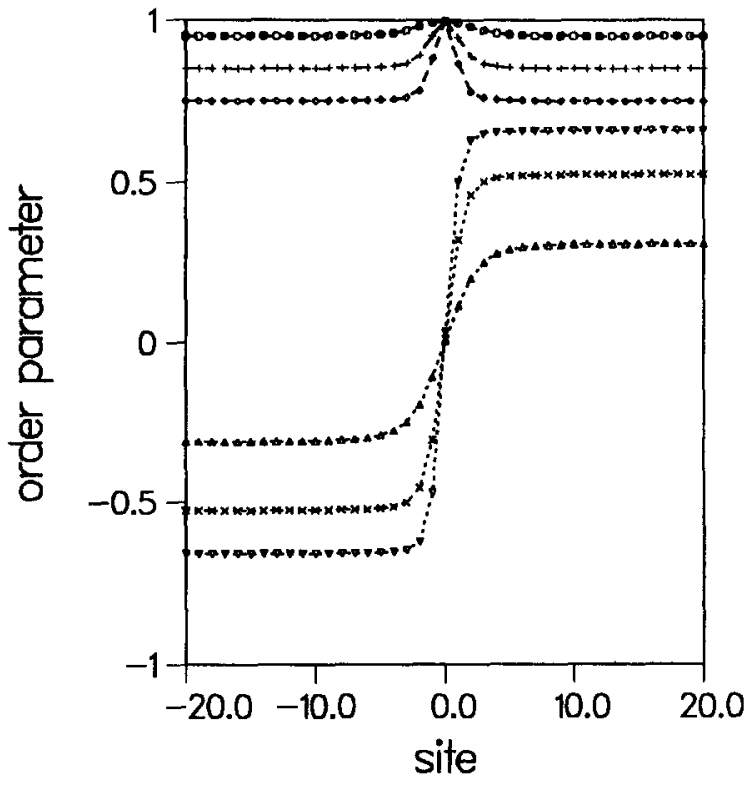

FIGURE 1

Charged vortex in 2D for $z J=4.0, B=3.0,3.4,3.8$; particle number $\left.\left(2<S^{2}\right\rangle=\cos \theta\right)$ : $\diamond,+$, o ; SC order parameter $\left(2<S_{\perp}>=\sin \theta\right): \nabla, \times, \Delta$.

The presence of charge in the vortices may have interesting consequences. For instance, it would give rise to a repulsive correction to the vortex-antivortex pair energy if long range Coulomb forces were added to the Hubbard model. In addition, a natural pinning mechanism for vortices offers itself: oppositely charged impurities, such as are present in the doped oxide superconductors, could bind the vortices electrostatically. This could be very effective since the mass of the vortex is estimated to be rather large.

\section{REFERENCES}

1. C.M. Varma, Phys. Rev. Lett. 61 (1988), 2713.

2. A.R. Bishop, P.S. Lomdahl, J.R. Schrieffer, and S.A. Trugman, Phys. Rev. Lett. 61 (1988), 2709.

3. L.J. de Jongh, in: Proceedings First International Symposium on Superconductivity, Nagoya, Japan, 1988 (Springer, Berlin, to be published).

4. R.T. Scalettar et al., Phys. Rev. Lett. 62 (1989), 1407.

5. S. Robaszkiewicz, R. Micnas, and K.A. Chao, Phys. Rev. B 23 (1981), 1447.

6. L.F. Feiner and J. Zaanen, to be published. 